\title{
Current practices for the genetic diagnosis of autoinflammatory diseases: results of a European Molecular Genetics Quality Network Survey
}

\author{
Dorota Rowczenio $^{1} \cdot$ Yael Shinar $^{2} \cdot$ Isabella Ceccherini $^{3} \cdot$ Katie Sheils $^{4} \cdot$ Marielle Van Gijn $^{5} \cdot$ Simon J. Patton $^{4}$. \\ Isabelle Touitou (10 ${ }^{6}$
}

Received: 21 February 2019 / Revised: 13 May 2019 / Accepted: 21 May 2019 / Published online: 11 June 2019

(c) The Author(s), under exclusive licence to European Society of Human Genetics 2019

\begin{abstract}
Monogenic autoinflammatory disorders (AIDs) are rare diseases caused by variants in genes regulating the innate immune system. The identification of the first four genes responsible for the prototype group of hereditary recurrent fevers prompted the development of genetic diagnosis, followed by external quality assessment and guidelines for the interpretation of sequence variants in these diseases. Recent changes in the diagnosis of genetic diseases, namely the implementation of nextgeneration sequencing (NGS), lead to discovery of the new genes associated with at least 40 novel AIDs, which revolutionized patient care and prognosis. However, these rapid advances resulted in nonstandardized molecular strategies that can influence genetic diagnosis and reporting of results. In order to assess factors, which may have an impact on performance and quality of results in the NGS era, we carried out an online survey among member laboratories of the European Molecular Genetics Quality Network, which highlighted different strategies being used and identified pitfalls that deserve discussion and improvement.
\end{abstract}

\section{Introduction}

Monogenic autoinflammatory disorders (AIDs) are rare diseases caused by molecular defects in genes involved in

These authors contributed equally: Dorota Rowczenio, Yael Shinar

Isabelle Touitou

Isabelle.touitou@inserm.fr

1 National Amyloidosis Centre, Division of Medicine, UCL, Royal Free Hospital, London, UK

2 Laboratory of FMF, Amyloidosis and Rare Autoinflammatory Diseases, Sheba Medical Center, Tel Hashomer, Israel

3 UOC Medical Genetics, Giannina Gaslini Institute, Genova, Italy

4 European Molecular Genetics Quality Network (EMQN), Manchester Centre for Genomic Medicine, St Mary's Hospital, Manchester M13 9WL, UK

5 Department of Genetics, University Medical Center Utrecht, Utrecht, Netherlands

6 INSERM, Department of Medical Genetics, Rare Diseases and Personalized Medicine, University of Montpellier, CEREMAIA, CHU Montpellier, Montpellier, France the regulation of innate immunity [1]. Patients have unprovoked recurrent episodes of inflammation including fever, abdominal, articular, and cutaneous signs with variable intensity and location. These symptoms may lack specificity making a clinical diagnosis difficult. Genetic testing provides early and accurate diagnosis and leads to appropriate treatment. AIDs were initially discovered in patients with hereditary recurrent fevers (HRF) i.e. familial Mediterranean fever (FMF) [2, 3], TNF receptor-associated periodic disease (TRAPS) [4], mevalonate kinase deficiency (MKD) [5] and cryopyrin-associated periodic diseases (CAPS) [6], and until recently genetic testing focused on these disorders.

Diagnostic genetic testing requires quality control procedures to ensure consistency and accuracy of results. An "in house" external quality assessment (EQA) scheme for the molecular diagnosis of HRF was set up in 2005 [7]. This study revealed that the harmonization of genetic testing procedures with standardized protocols was essential for laboratories to achieve a minimum level of expertise and ensure appropriate patient care. The European Molecular Genetics Quality Network (EMQN) was established in 1998 and originally was supported by a grant from the European Commission. Since then, EMQN have developed their 
scope of EQAs and the HRF scheme was introduced in 2009 as part of the EMQN EQA portfolio. In 2012, we proposed initial guidelines for the genetic diagnosis of HRF [8]. In 2015, we provided clinical utility gene cards for the four prototypic HRF syndromes [9].

Today, more than 40 monogenic disorders have been characterized affecting different components of the innate immune system [10]. In addition, laboratory practices are changing rapidly due to the implementation of next-generation sequencing (NGS) technologies and an increased demand for DNA screening in the era of treatment-oriented genetic testing.

To assess the current trends in genetic tests for AIDs, we organized an online survey whose objective was to collect critical information on current practices to identify cardinal problems (e.g., bottlenecks in the referrals for AIDs genetic testing, laboratory workload problems, technical issues or ethics limitations), and to propose solutions, which may be adapted by any laboratory performing AIDs genetic testing. Herein, we report the results of this study, and discuss them in the light of past practices and future directions and guidelines.

\section{Materials and methods}

\section{Organization of a preparatory workshop}

We took the opportunity at the 52nd conference of the European Society of Human Genetics (Milan, June 2018) to organize a small meeting attended by geneticists from different countries carrying out AIDs diagnostic tests. The debate was not restricted to specific diseases as genetic testing for this group of disorders varies worldwide. After several rounds of discussions, the elements for collecting of information relevant for our objectives, such as current legislation, any prerequisites required, clinical forms, techniques used, quality and performance advice were selected.

\section{Definition of the survey components}

We were aware that some laboratories require specific information or analyses before accepting samples for genetic testing as administrative requirements differ from country to country and given the growing demand for genetic diagnosis. We therefore designed a concise survey of six questions grouped into three main themes: preconditions for genetic testing (to assess both administrative, except for specific legal issues, i.e. prescription by a physician and patient consent, and clinical data that may be useful in the interpretation of results), managing of asymptomatic individuals (feedback regarding testing of asymptomatic relatives) and sequencing strategy (to assess the extent of implementation of new DNA sequencing strategies among laboratories) (Table 1).
Table 1 Items of the survey

Questions Answer choices

Your current prerequisites

Q1: Do you have any administrative prerequisites apart from informed consent and prescription?

Yes (If yes, please specify below:)

No

Other (please specify)

Q2: Does your organization require any of the following medical prerequisites:

Genealogic tree

Elevated CRP or leukocytosis during/between attacks

Age at disease onset (if limit please define in y)

Minimal number recurrent attacks $\geq 3$

Exclusion of other disease(s)

Ancestry

Mevalonic aciduria (MKD)

ADA2 enzymatic defect (DADA2)

Other (clinical suspicion or phenotype description for example)

Q3: Does your organisation test asymptomatic individuals (according to your laws and practice)?

Never performed

Performed only for adults (not allowed in children)

Performed only for seggregation analysis, never reported

Performed and reported only if specifically ordered

Performed and reported always

Other (please specify)

Your current technical approach

Q4: What is your mutation analysis strategy?

Non-NGS

Only panels

Only WES

Both NGS and non-NGS depending on the clinician request

Both NGS and non-NGS depending on my own choice

Somatic mutation testing

Other

Q5: What is your current mutation validation approach?

Depends on the technique quality

Systematic second technique

Systematic parent analysis

Identito-vigilance

Other

Q6: Do you have any suggestions for a better efficiency of testing?

DMSO for GC-rich regions

Other (please specify) 


\section{Diffusion of the survey}

We reasoned that the survey should be brief, user-friendly and that the questions specific enough to motivate the participants. Multiple-choice questions alongside a small number of open-ended questions were used. A web-based questionnaire hosted on Survey Monkey was set up to survey the EMQN laboratories participating in the HRF EQA scheme. The url link to the survey was distributed by email three times to all EMQN HRF-scheme participants during the summer of 2018 (July and August).

\section{Results}

\section{Participant profiles}

All 90 participants of the EMQN HRF-scheme from 2009 until present were invited to participate in the survey. We obtained responses from 23 laboratories (19 from 10 different European countries, and one from the USA, New Zealand, Australia and Brazil), which consisted of a mixture of private and public institutions. The raw data were extracted as an excel file on August 22, 2018, and the results interpreted.

\section{Responses in detail}

\section{Test prerequisites}

The results are presented in Fig. 1. Most laboratories (18/23, $78 \%$ ) do not have specific administrative requirements. Two participants mentioned financial issues, one analyzed all samples ordered by the treating physician, and two commented on the content of the clinical form and consent. As for the medical prerequisites, half of the laboratories reported that they do not request specific medical information. No clinical pre-requisite seemed to reach a consensus or majority of votes. Among the preferred medical information requested were: pediatric age at disease onset (38\%), evidence of biological inflammation (33\%), family tree (29\%), and ancestry (24\%). We established that enzyme tests for the two AIDs, although very indicative, were barely (MKD), or not requested at all (adenosine deaminase 2 deficiency, DADA2) before undertaking genetic testing.

\section{Testing of asymptomatic individuals}

The results are presented in Fig. 2. Half of the laboratories only performed and reported such analyses if asked to do so by the clinician. Two stated that genetic counseling should always precede the testing of asymptomatic individuals. Two participants indicated that they adapt their procedure to specific clinical conditions. One suggested that siblings of index cases should be tested regardless of age and clinical status (healthy or affected) in severe diseases. One such example mentioned was DADA2 because of the high risk of a stroke in presymptomatic individuals.

\section{Technical approach}

The results are presented in Fig. 3. Sequencing of gene panels using NGS platforms is now the preferred strategy for AIDs. Six laboratories use Sanger sequencing as a sole method of DNA analysis, but one of these is currently transitioning to NGS. No other approaches are used as a first-line testing strategy-this is a seismic change in AIDs diagnostic practice since the first guidelines were published [8].

Typically, 67\% (14/21) laboratories did not systematically validate DNA results using an orthogonal method. Participants reported that this practice depended primarily on the molecular geneticist's assessment of the technical quality of the test result. Of the laboratories that did validate the variant(s) found, Sanger sequencing was used by all, except one that used restriction fragment length polymorphism. One laboratory had developed deep sequencing $(1000-10,000 \times)$ for postzygotic variants. Parental testing was not systematically performed but was considered useful for variant phasing in recessive disorders. Identity testing was rarely undertaken $(3 / 21,14 \%)$.

Finally, we evaluated the suggestions for better efficiency (not tabulated). This last query was an open-ended, free-text question, with only one suggestion related to the difficulty of sequencing through GC-rich sequences (such as $M E F V$ exon 2 for example). Thirty-three per cent (7/21) of the laboratories acknowledged the importance of using dimethyl sulphoxide to help examine such regions. One participant suggested the use of different primers for amplification and sequencing, and another made clear recommendations on when to target specific genes, and the use of narrow or extended panels.

\section{Summary and proposed solutions}

The only pitfall of this survey was the difficulty to assess the multiple choices made by the participants. A number of common practices and issues could be highlighted from this survey, which are summarized in Table 2. We identified advantages and weaknesses in the NGS era, and proposed possible solutions for the six items investigated in our survey: notably a minimal core of medical information is required, a decision tree for testing strategy might be helpful when deciding on the method, and a wider use of AIDsexperts or networks. Moreover, the heterogeneous approach to DNA analysis prompted us to write an up-to-date best 

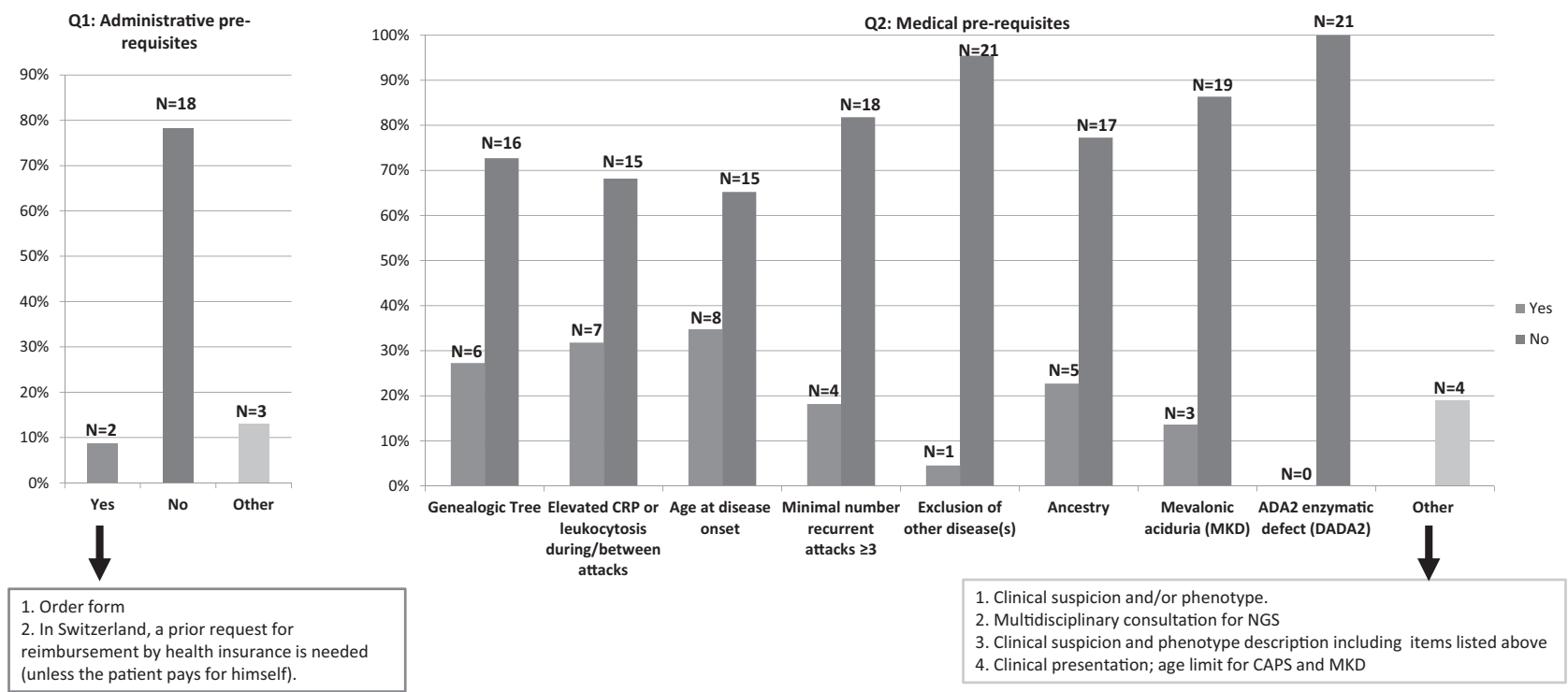

Fig. 1 Prerequisites for the genetic diagnosis of AID. On the left: Administrative prerequisites. The three responses "Other" were out of the scope of this question (see text) and are not mentioned here. On the right: Medical prerequisites. AID autoinflammatory disorder

Fig. 2 Testing of asymptomatic individuals. A nonuniform approach to test request and reporting procedures was found among the 23 participating laboratories

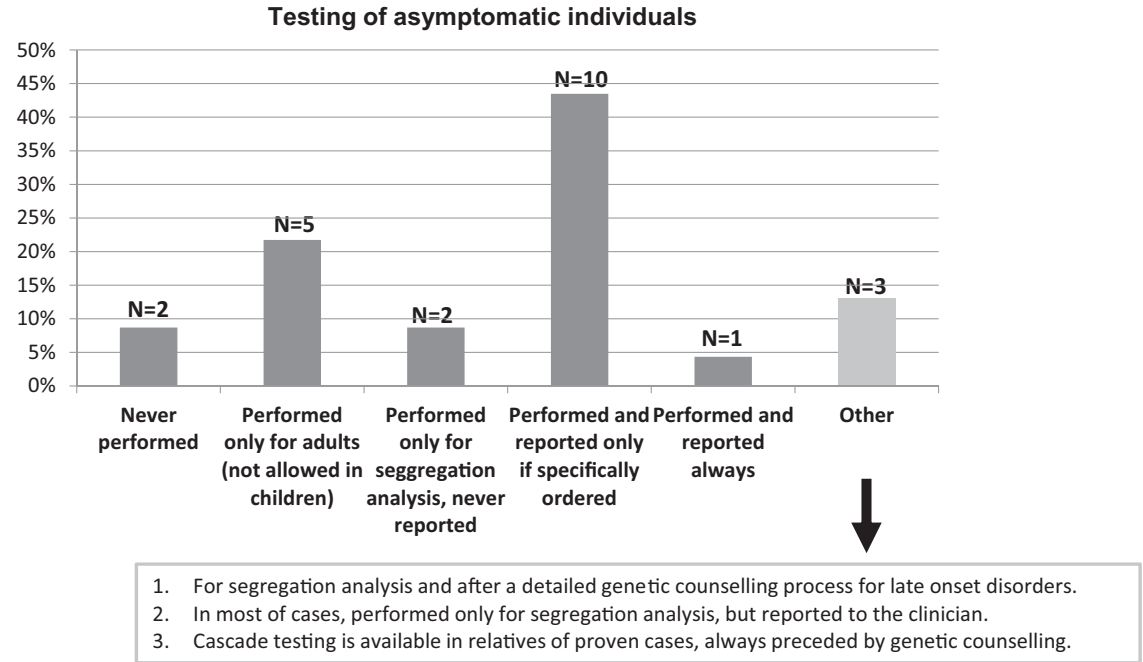

practice guidelines for genetic diagnostics in AIDs (manuscript in preparation).

\section{Discussion}

An online survey among the different genetic laboratories participating in the EMQN EQA scheme for HRF was performed, and their responses systematically analyzed and summarized in this manuscript. This survey revealed a transformation in diagnostic practices since the initial implementation of genetic testing for AIDs worldwide, first for FMF in 1997 [11], then upon each AID gene discovery, with an initial introduction of the EQA in 2005 [7], leading to establishment of the best practice guidelines for HRF in
2012 [8]. The main advance was the implementation of the NGS method by most laboratories. The survey also identified trends in test prerequisites and case processing that are worth discussing in the NGS era.

The administrative prerequisites for genetic testing of this group of disorders have not changed over the years. This study shows that there is also no consensus on medical prerequisites. This is surprising because information that may influence the choice of the genetic analysis or the interpretation of the DNA results were not available to most laboratories performing the genetic testing. For instance, having biological evidence of inflammation before undertaking a genetic test increases the chances of effectively targeting AIDs. However, CRP values during attacks were not requested by two thirds of the respondent laboratories. Enzymatic testing for certain AIDs is 
Q4. Variant analysis strategy

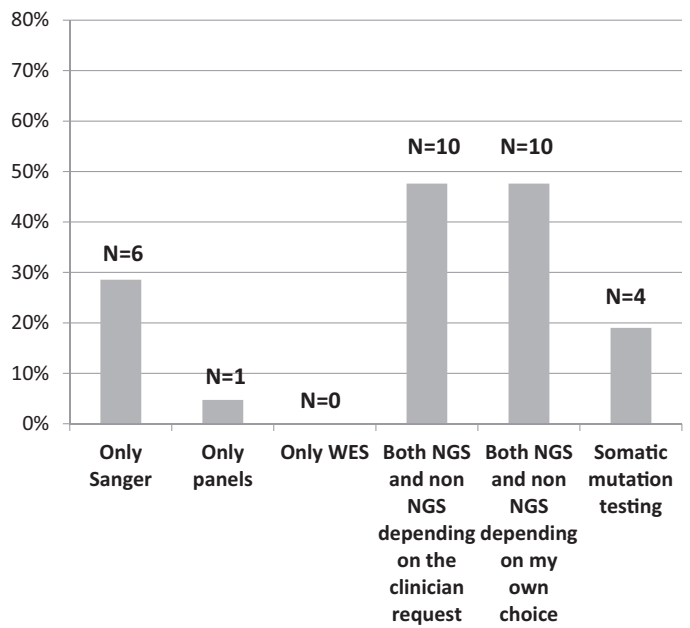

Q5. Validation strategy

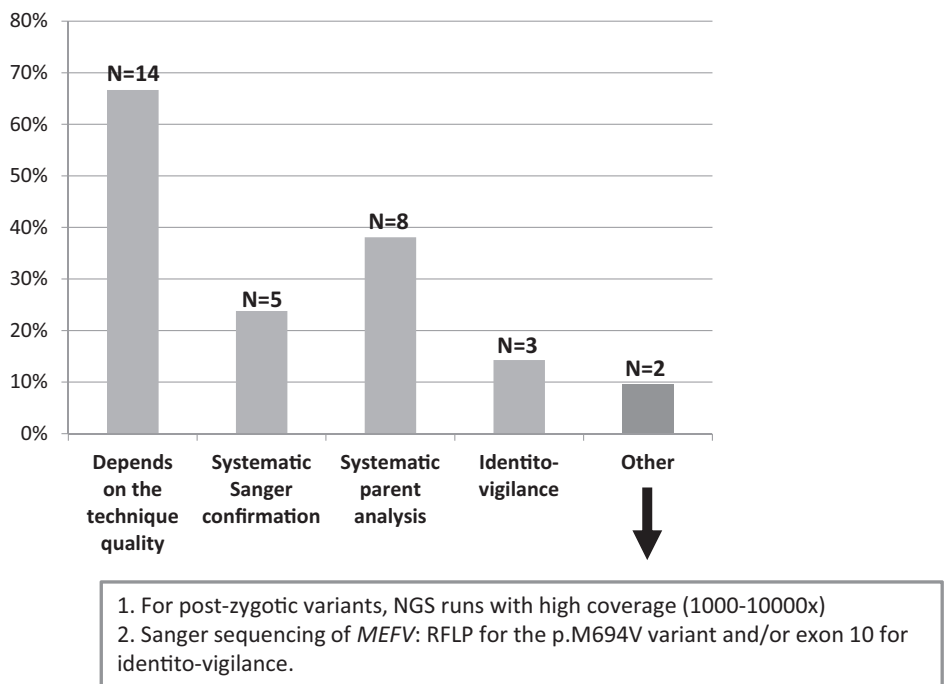

Fig. 3 Technical approach used. On the left: DNA analysis strategy. On the right: Validation of DNA results strategy. The total number of responses exceeds the number of participants because it is a multichoice question

also possible; however, the respondents indicated that this was not commonly requested. Possible reasons might be that measurements for the ADA2 enzyme activity are not widely available, and that the pathophysiology of DADA2 is still unclear [12]. On the other hand, the mevalonic aciduria assay is considered a good biomarker for MKD, but this test is infrequently requested [13].

Most laboratories do not have information on age of onset and family history, while it has recently been discovered that somatic variants can cause AIDs [14]. This is an important finding since about $40 \%$ of respondents reported that they have a choice over the method they use for testing. In addition, the interpretation of DNA variants is largely based on the allele frequency established for the general population, which can lead to misinterpretation of genetic findings. Thus, having the information on the patient's ancestry would help to determine the rarity of a given variant in a specific ethnic group through public databases like gnomAD (http://gnomad.broadinstitute.org/).

The reasons for the lack of consensus on medical prerequisites, or rather general lack of medical prerequisites is probably due to a combination of the following: the medical doctors not recognizing the need to provide such information, the differences in laboratory background, i.e. whether they are general genetic or disease expert laboratories and/ or implementation of the national strategies for genetic testing.

Testing of asymptomatic individuals varied considerably between the respondent laboratories. However, familial studies are essential for phasing variants, determining if the variant occurred de novo, or helping to extract the clinical relevance in a variant of unknown clinical significance. In those cases, over diagnosis is a risk if segregation analysis is not performed.

NGS was the preferred technical approach to testing, implemented by the majority of laboratories. The survey has identified procedures that could be improved, or at least homogenized, for example testing for somatic variants (currently undertaken by only $17 \%$ of the respondent laboratories). Most laboratories do not perform Sanger validation of NGS findings. When performed, no systematic strategy emerged from the results returned to us. Rather, the decision to validate the DNA results depended on the technical quality of the approach (horizontal and vertical coverage of the target sequence) and on the complexity of the variants detected. Another reason for choosing the Sanger confirmation is sample traceability to avoid sample mix-ups.

Previous reports highlighted a low diagnostic yield $(<30 \%)$ in the patients with undifferentiated AIDs who underwent NGS panel testing [15-17], but one cannot conclude that the problems identified in this survey are responsible for this low performance.

In conclusion, there has been a seismic change in the technical approaches used for testing in this group of disorders since the first guidelines in 2012 [8], with move towards high-throughput sequencing, and away from other DNA testing procedures. This survey implied that currently there is a heterogeneous approach used in the variant analyses for the AIDs, which can affect both the quality of testing and the reporting of the results. Our findings are relevant not only to AIDs but to NGS genetic testing in general, and sustain the need to revise the current 2012 AIDs guidelines to drive 


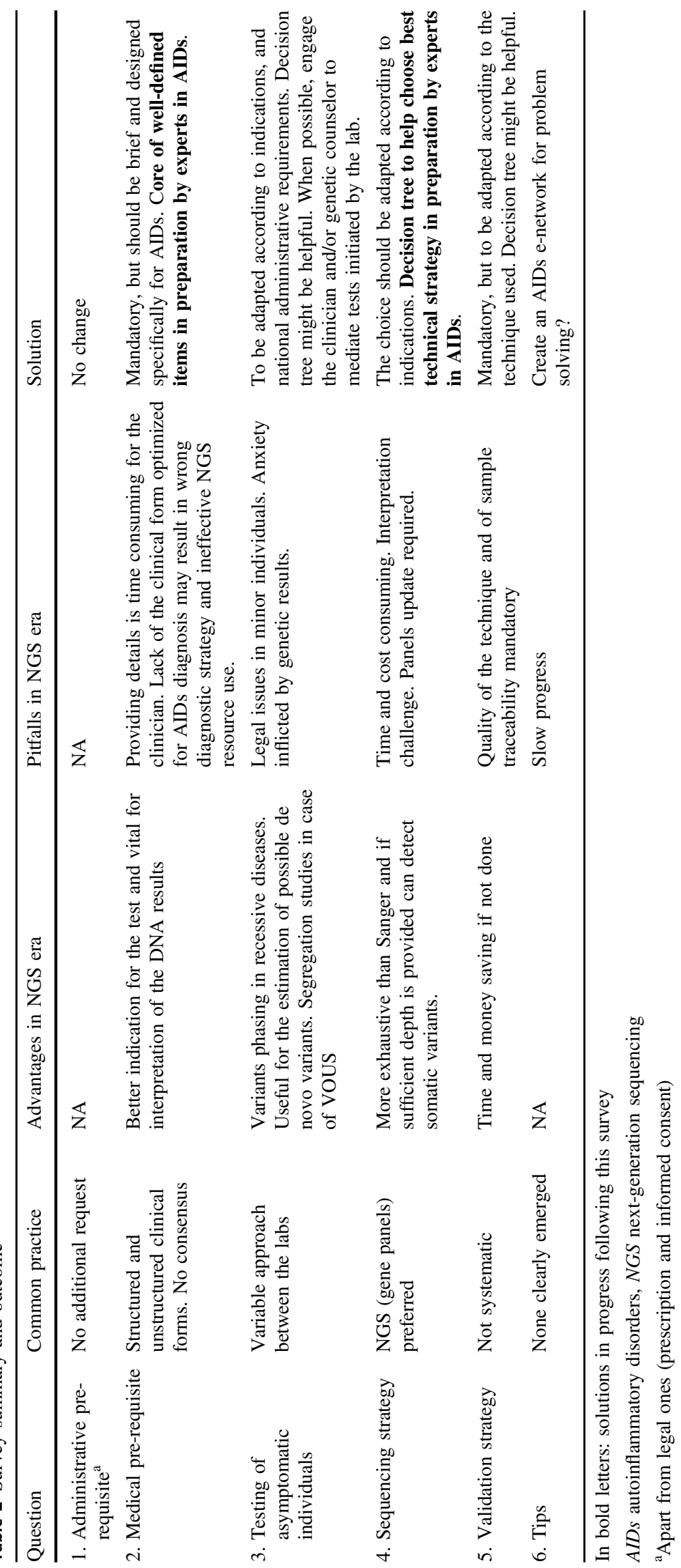


improvements in laboratory testing practice (manuscript in preparation).

Acknowledgements The authors thank the other attendees of the preparatory meeting in Milan (Drs. Guilaine Boursier, Sergio Burillo, Stefan Nemeth, Christian Oberkanans, Lennart Opitz, Tamara Sarkizian, Nicola Wolstenholme, and Evelien Zonneveld-Huijssoon) for fruitful discussions.

\section{Compliance with ethical standards}

Conflict of interest The authors declare that they have no conflict of interest.

Publisher's note: Springer Nature remains neutral with regard to jurisdictional claims in published maps and institutional affiliations.

\section{References}

1. Manthiram K, Zhou Q, Aksentijevich I, Kastner DL. The monogenic autoinflammatory diseases define new pathways in human innate immunity and inflammation. Nat Immunol. 2017;18:832-42.

2. French FMF Consortium. A candidate gene for familial Mediterranean fever. Nat Genet. 1997;17:25-31.

3. The International FMF Consortium. Ancient missense mutations in a new member of the RoRet gene family are likely to cause familial Mediterranean fever.Cell. 1997;90:797-807.

4. McDermott MF, Aksentijevich I, Galon J, McDermott EM, Ogunkolade BW, Centola M, et al. Germline mutations in the extracellular domains of the $55 \mathrm{kDa}$ TNF receptor, TNFR1, define a family of dominantly inherited autoinflammatory syndromes. Cell. 1999;97:133-44.

5. Drenth JP, Cuisset L, Grateau G, Vasseur C, van de Velde-Visser $\mathrm{SD}$, de Jong JG, et al. Mutations in the gene encoding mevalonate kinase cause hyper-IgD and periodic fever syndrome. International Hyper-IgD Study Group. Nat Genet. 1999;22:178-81.

6. Hoffman HM, Mueller JL, Broide DH, Wanderer AA, Kolodner RD. Mutation of a new gene encoding a putative pyrin-like protein causes familial cold autoinflammatory syndrome and Muckle-Wells syndrome. Nat Genet. 2001;29:301-5.
7. Touitou I, Rittore C, Philibert L, Yagüe J, Shinar Y, Aksentijevich I. An international external quality assessment for molecular diagnosis of hereditary recurrent fevers: a 3-year scheme demonstrates the need for improvement. Eur J Hum Genet. 2009;17:890-6.

8. Shinar Y, Obici L, Aksentijevich I, Bennetts B, Austrup F, Ceccherini I, et al. Guidelines for the genetic diagnosis of hereditary recurrent fevers. Ann Rheum Dis. 2012;71:1599-605.

9. Witsch-Baumgartner M, Touitou I. Clinical utility gene card for: prototypic hereditary recurrent fever syndromes (monogenic autoinflammatory syndromes). Eur J Hum Genet. 2015;23:https:// doi.org/10.1038/ejhg.2014.257.

10. Martinon F, Aksentijevich I. New players driving inflammation in monogenic autoinflammatory diseases. Nat Rev Rheuma. 2015;11:11-20.

11. Dupont M, Dross C, Smaoui N, Nedelec B, Grateau G, Clépet C, et al. Genotypic diagnosis of familial Mediterranean fever (FMF) using new microsatellite markers: example of two extensive nonAshkenazi Jewish pedigrees. J Med Genet. 1997;34:375-81.

12. Caorsi R, Penco F, Grossi A, Insalaco A, Omenetti A, Alessio M, et al. ADA2 deficiency (DADA2) as an unrecognised cause of early onset polyarteritis nodosa and stroke: a multicentre national study. Ann Rheum Dis. 2017;76:1648-56.

13. Jeyaratnam J, Ter Haar NM, de Sain-van der Velden MGM, Waterham HR, van Gijn ME, Frenkel J. Diagnostic value of urinary mevalonic acid excretion in patients with a clinical suspicion of mevalonate kinase deficiency (MKD). JIMD Rep. 2016;27:33-8.

14. Labrousse M, Kevorkian-Verguet C, Boursier G, Rowczenio D, Maurier F, Lazaro E, et al. Mosaicism in autoinflammatory diseases: cryopyrin-associated periodic syndromes (CAPS) and beyond. A systematic review. Crit Rev Clin Lab Sci. 2018;55:432-42.

15. Nakayama M, Oda H, Nakagawa K, Yasumi T, Kawai T, Izawa $\mathrm{K}$, et al. Accurate clinical genetic testing for autoinflammatory diseases using the next-generation sequencing platform MiSeq. Biochem Biophys Rep. 2017;9:146-52.

16. Omoyinmi E, Standing A, Keylock A, Price-Kuehne F, Melo Gomes S, Rowczenio D, et al. Clinical impact of a targeted nextgeneration sequencing gene panel for autoinflammation and vasculitis. PLoS ONE. 2017;12:e0181874.

17. Rusmini M, Federici S, Caroli F, Grossi A, Baldi M, Obici L, et al. Next-generation sequencing and its initial applications for molecular diagnosis of systemic auto-inflammatory diseases. Ann Rheum Dis. 2016;75:1550-7. 